\title{
Correção do astig̉matismo na cirurgia da catarata
}

\author{
Surgical correction of astigmatism duringcataract surgery
}

\author{
Edison Ferreira e Silva ${ }^{1}$ \\ Fernando Cançado Trindade ${ }^{2}$
}

Trabalho realizado na Oftalmoclínica Icaraí - Niterói - RJ

${ }^{1}$ Doutor em Oftalmologia; Diretor Oftalmoclínica Icaraí, Niterói (RJ) - Brasil.

${ }^{2}$ Doutor em Oftalmologia; Chefe do Serviço de Córnea, Catarata, Doenças Externas Oculares e Cirurgia Refrativa do Hospital São Geraldo - Belo Horizonte (MG) - Brasil.

Endereço para correspondência: Edison Ferreira e Silva. Rua Miguel de Frias, 40 - $8^{\circ}$ Andar - Niterói (RJ) CEP 24220-002

E-mail: edis98@uol.com.br

Recebido para publicação em 15.09.2006

Última versão recebida em 04.05.2007

Aprovação em 21.05.2007

\section{RESUMO}

Objetivos: Avaliar a eficácia das incisões periféricas relaxantes limbares (IPRL) na redução do astigmatismo pré-operatório durante a cirurgia de catarata. Métodos: Foram estudados prospectivamente 103 olhos de 103 pacientes submetidos as IPRL, utilizando o nomograna de Nichamin durante a cirurgia de catarata pela facoemulsificação. Após o $1^{\circ}$ e $6^{\circ}$ mês foram avaliadas as mudanças no astigmatismo topográfico, na indução do astigmatismo e no índice de sucesso. Os pacientes foram separados em dois grupos segundo o tipo de astigmatismo no pré-operatório (a favor da regra e contra a regra) e estudados separadamente. Resultados: Ocorreram diferenças estatisticamente significativas entre os valores dos astigmatismos topográficos no pré e pós-operatório nos dois grupos. Verificou-se indução de $1,10 \pm 0,9$ dioptrias e $37 \%$ de índice de sucesso no grupo de astigmatismo a favor da regra e $1,70 \pm 0,80$ dioptrias e $51 \%$ de índice de sucesso no grupo de astigmatismo contra a regra após o $6^{\circ}$ mês de seguimento. Conclusão: A incisão periférica relaxante limbar é efetiva na redução do astigmatismo pré-existente durante a cirurgia da catarata. O procedimento mostrou ser seguro e de fácil realização. O nomograma de Nichamim na nossa experiência hipocorrige o astigmatismo planejado em ambos os grupos estudados.

Descritores: Astigmatismo; Astigmatismo/cirurgia; Facoemulsificação; Extração de catarata

\section{INTRODUÇÃO}

A cirurgia da catarata passou a ser considerada tanto para o paciente como para o cirurgião uma cirurgia refracional. Com os implantes intraoculares esfericamente perfeitos, a superfície anterior da córnea é o único local responsável pela presença de astigmatismo no pós-operatório. O astigmatismo presente após a cirurgia pode ser conseqüência de astigmatismo congênito ou a indução causada pela própria cirurgia.

Meios diagnósticos precisos como a ceratometria automatizada e a topografia computadorizada ${ }^{(1)}$, nos permite avaliação prévia da superfície da córnea tanto quantitativa como qualitativamente, identificando alterações pré-existentes. A utilização cada vez maior de implantes intra-oculares multifocais fica em muitos casos impossibilitada devido a astigmatismo superior a uma dioptria, privando o paciente de uma recuperação visual completa (longe e perto).

Dentre as opções para a redução do astigmatismo ${ }^{(2-6)}$, a técnica incisional corneana realizada no momento da cirurgia da catarata se mostrou eficaz na aplanação do meridiano mais curvo da córnea. Entre as técnicas utilizadas, a incisão periférica relaxante limbar (IPRL) é uma opção segura e de simples execução para a redução do astigmatismo pré-existente. Essa técnica tem por base a utilização do grau de astigmatismo pré-existente e a idade dos pacientes, relacionados na forma de um nomograma para orientar as incisões, entre eles o de Nichamin ${ }^{(7-8)}$. 
O objetivo desse trabalho é avaliar a eficácia da técnica IPRL na redução do astigmatismo corneano pré-existente utilizando-se o nomograma de Nichamin durante a cirurgia de catarata pela facoemulsificação.

\section{MÉTODOS}

Estudamos prospectivamente 123 pacientes eleitos para a cirurgia de catarata pela técnica de facoemulsificação que apresentavam no pré-operatório astigmatismo corneano $\geq 1,00 \mathrm{D}$. Os pacientes foram submetidos ao exame oftalmológico completo, incluindo: paquimetria ultra-sônica, ceratoscopia computadorizada (Atlas-Humphrey-Zeiss ${ }^{\mathrm{TM}}$ ) e ceratometria automática (Humphrey-Zeiss ${ }^{\mathrm{TM}}$ ). Foram excluídos do estudo pacientes que apresentavam astigmatismo irregular ao exame da ceratoscopia computadorizada, presença de pterígeo, cirurgia do segmento anterior prévia, trauma corneano e qualquer patologia corneana superficial. Todos os pacientes foram submetidos à cirurgia de catarata no período de janeiro de 2003 a dezembro de 2004. O estudo foi aceito pelo conselho de ética da Universidade Federal do Estado de Minas Gerais, Brasil. Os pacientes leram e assinaram o consentimento informado sobre a cirurgia a ser realizada concomitantemente com a cirurgia da catarata.

Dos 123 pacientes operados 20 pacientes foram excluídos do trabalho por: não cumprirem as visitas de $1^{\circ} \mathrm{e} / \mathrm{ou} 6^{\circ}$ mês (16 pacientes) e por falecimento antes do $6^{\circ}$ mês (4 pacientes).

Todas as cirurgias foram realizadas pelo mesmo cirurgião (EFS), utilizando a mesma técnica cirúrgica. Realizamos $60 \%$ das cirurgias sob anestesia tópica e sedação e no restante utilizamos bloqueio peribulbar e sedação. Os pacientes foram submetidos à cirurgia de incisão relaxante limbar no início da cirurgia de facoemulsificação seguindo o nomograma e técnica descrita por Nichamin ${ }^{(8)}$. O número e tamanho das incisões foram realizados seguindo o nomograna baseado na idade e dioptrias de astigmatismo pré-existente medido pela ceratoscopia computadorizada. Para minimizar o efeito rotacional, marcas no limbo foram colocadas antes da cirurgia na posição de 180 graus com o paciente sentado, obtendo-se assim o correto eixo incisional. As incisões foram realizadas na parte interna da arcada vascular limbar, utilizando bisturi de diamante calibrado em $600 \mu$ de profundidade. As incisões foram realizadas ao longo do meridiano mais curvo, determinado pela ceratoscopia computadorizada. Todos os olhos receberam pares de incisões, opostas 180 graus.

A facoemulsificação foi realizada por incisão corneana descrita por I. H. Fine ${ }^{(9)}$, com paracentese da câmara anterior com bisturi de diamante de 30 graus de $0,5 \mathrm{~mm}$ de largura localizada entre 60 a 80 graus de distância da incisão principal. A incisão corneana principal foi realizada em todos os pacientes no meridiano de 180 temporal sendo seu desenho biplanar, retangular de 2,8 x 2,0 mm, utilizando bisturi de diamante de 2,8 $\mathrm{mm}$ de largura. A facoemulsificação do núcleo foi endocapsular com ponteiras de 2,6 $\mathrm{mm}$. O implante da lente intra-ocular (Alcon
Acrysof SA e SNTM e Sensar AMO ${ }^{\mathrm{TM}}$ ) ocorreu sem o aumento da incisão através do uso de injetor. Todas as LIOs foram implantadas no saco capsular com as alças na posição horizontal. Todas as incisões foram auto-selantes sem sutura.

Os medicamentos pós-operatórios utilizados foram: quinolonas de quarta geração por 10 dias e prednisolona $0,1 \% \mathrm{com}$ diminuição gradativa por um período de 30 dias.

Os pacientes foram divididos em dois grupos no pré-operatório: astigmatismo a favor da regra (meridiano mais curvo compreendido entre 46 e 135 graus) e contra a regra (meridiano mais curvo compreendido entre zero e 45 graus e 136 e 180 graus).

Para acompanhamento da indução do astigmatismo utilizamos a topografia computadorizada no $1^{\circ}$ mês e no $6^{\circ}$ mês do pós-operatório.

Analisamos os seguintes tópicos:

1. Média aritmética das mudanças do astigmatismo topográfico

2. Determinação da indução do astigmatismo pelas incisões relaxantes limbares

3. Análise agregada dos astigmatismos topográficos e indução

4. Regressão linear correlacionando astigmatismo e idade

5. Índice de sucesso

Utilizamos o método de Alpins ${ }^{(10-11)}$ para o cálculo da indução do astigmatismo e o índice de sucesso no pós-operatório e comparando-os entre o $1^{\circ}$ e $6^{\circ}$ mês de cirurgia em cada grupo. A análise agregada dos astigmatismos topográficos foi calculada a partir dos trabalhos de Holladay ${ }^{(12)}$.

Utilizamos o teste $\mathrm{t}$ de Student e a regressão linear para as análises estatísticas com nível de significância de 5\%.

\section{RESULTADOS}

Do total de 123 pacientes, 103 compareceram aos dois exames de topografia computadorizada ( $1^{\circ}$ e $6^{\circ}$ mês), sendo então eleitos para participar do estudo.

O grupo a favor da regra foi composto de 41 pacientes, sendo 10 homens e 31 mulheres. A idade média do grupo foi de $70,5 \pm 6,7$ anos, variando entre 56 e 86 anos. O grupo contra a regra foi composto de 62 pacientes, sendo 22 homens e 40 mulheres. A idade média foi de 77,4 $\pm 7,6$ anos, variando entre 61 e 92 anos (Tabela 1).

O grupo a favor da regra apresentou astigmatismo médio no pré-operatório de $2,1 \pm 1,0$ dioptrias. Após o $1^{\circ}$ mês apresentou astigmatismo médio de 1,3 $\pm 0,6$ e indução média de $1,1 \pm 0,8$ dioptrias e no $6^{\circ}$ mês apresentou astigmatismo médio de $1,4 \pm 0,8$

\begin{tabular}{|lcc|}
\hline \multicolumn{3}{|c|}{ Tabela 1. Distribuição demográfica dos pacientes } \\
\cline { 2 - 3 } & \multicolumn{2}{c|}{$\mathbf{n = 1 0 3}$} \\
\cline { 2 - 3 } Idade & AFR & CR \\
Homens & $11(27 \%)$ & $77,4 \pm 7,6(61-92)$ \\
Mulheres & $30(73 \%)$ & $22(35)$ \\
AFC= a favor da regra; CR= contra a regra & $40(65)$ \\
\hline
\end{tabular}


e indução média de 1,1 $\pm 0,9$ dioptrias. $O$ índice de sucesso foi de $0,64 \pm 0,2(36 \%)$ no $1^{\circ}$ mês e $0,63 \pm 0,2(37 \%)$ no $6^{\circ}$ mês. Comparando o astigmatismo topográfico do pré-operatório com aqueles do $1^{\circ}$ e $6^{\circ}$ mês encontramos diferenças estatisticamente significativas nos dois casos $(\mathrm{p}<0,0001)$. Comparando a indução e o índice de sucesso entre o $1^{\circ}$ e $6^{\circ}$ mês não encontramos diferenças estatisticamente significativas (Tabela 2). $\mathrm{Na}$ regressão linear o astigmatismo induzido e a idade, não apresentaram associação estatisticamente significativa (coeficiente de regressão $=0,0117$ com IC $95 \%-0,029$ a 0,052). O astigmatismo agregado foi de $1,81 \pm 1,51 \mathrm{D} @ 97^{\circ}$ no préoperatório e $1,08 \pm 1,20 \mathrm{D} @ 101^{\circ}$ no pós-operatório após o $6^{\circ}$ mês (Figura 1). A diferença de ângulo entre o pré e o pósoperatório foi de 4 graus.

O grupo contra a regra apresentou astigmatismo médio no pré-operatório de 2,2 $\pm 0,9$ dioptrias. Após o $1^{\circ}$ mês apresentou astigmatismo médio de $1,1 \pm 0,7$ e indução média de $1,9 \pm$ 0,9 e no $6^{\circ}$ mês apresentou astigmatismo médio de $1,1 \pm 0,7$ e indução média de $1,7 \pm 0,8$ dioptrias. $O$ índice de sucesso foi de $0,50 \pm 0,2(50 \%)$ no $1^{\circ}$ mês e $0,49 \pm 0,2(51 \%)$ no $6^{\circ}$ mês. Comparando o astigmatismo topográfico do pré-operatório com aqueles do $1^{\circ}$ e $6^{\circ}$ mês encontramos diferenças estatisticamente significativas nos dois casos $(\mathrm{p}<0,0001)$. Comparando a indução e o índice de sucesso entre o $1^{\circ}$ e $6^{\circ}$ mês não encontramos diferenças estatisticamente significativas (Tabela 3). $\mathrm{Na}$ regressão linear o astigmatismo induzido e a idade, não apresentaram associação estatisticamente significativa (coeficiente de regressão $=0,077$ com IC $95 \%-0,019$ a 0,034). O astigmatismo agregado foi de $1,75 \pm 1,57 \mathrm{D} @ 6^{\circ}$ no préoperatório e de $0,48 \pm 1,21 \mathrm{D} @ 11^{\circ}$ no pós-operatório após o $6^{\circ}$ mês (Figura 2). A diferença de ângulo entre o pré e o pósoperatório foi de 5 graus.

Observando o número e a porcentagem de pacientes e a dioptria topográfica no pré-operatório verificamos que apenas $5,82 \%$ dos pacientes ficavam entre zero e uma dioptria e que após seis meses esse número se elevou para 50,48\% (Tabela 4).

Comparando os dois grupos encontramos diferenças estatisticamente significativas em relação à idade sendo o grupo contra a regra mais velho do que o a favor da regra $(\mathrm{p}<0,0001)$. O valor dióptrico topográfico inicial, após o $1^{\circ}$ e após o $6^{\circ}$ mês não apresentaram diferenças estatisticamente significativas entre os dois grupos $(p=0,86, p=0,11$ e $p=0,56)$. A indução e o

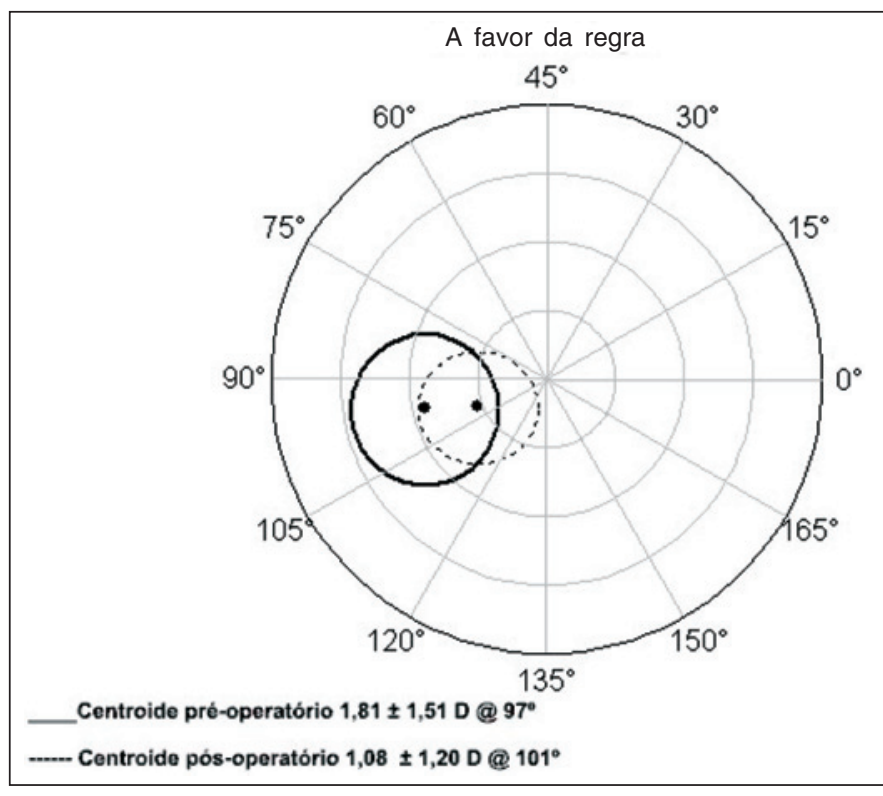

Figura 1 - (Cagy) Análise vetorial agregada do astigmatismo a favor da regra no pré e pós-operatório após o $6^{\circ}$ mês de acompanhamento. Os pontos escuros representam as médias e as elipses representam o desvio padrão. Cada anel do gráfico representa uma dioptria.

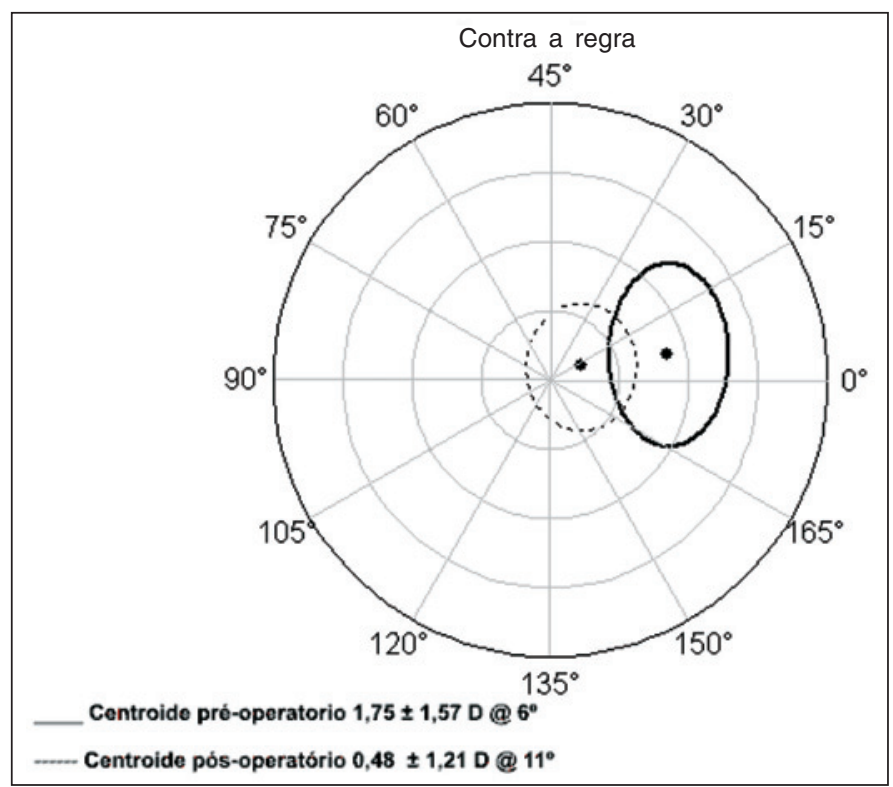

Figura 2 - (Cagy) Análise vetorial agregada do astigmatismo contra a regra no pré e pós-operatório após o $6^{\circ}$ mês de acompanhamento. Os pontos escuros representam as médias e as elipses representam o desvio padrão. Cada anel do gráfico representa uma dioptria.

\begin{tabular}{|c|c|c|c|c|}
\hline \multicolumn{5}{|c|}{ Tabela 2. Astigmatismo a favor da regra pré e pós-operatório $1^{\circ}$ e 60 mês } \\
\hline & \multicolumn{4}{|c|}{$n=41$} \\
\hline & Pré-operatório & Pós-1ำ mês & Pós- 6 mês & $p 1^{\circ} \times 6^{\circ}$ \\
\hline Astigmatismo & $2,1 \pm 1,0$ & $1,30 \pm 0,6$ & $1,40 \pm 0,8$ & 0,54 \\
\hline Indução & & $1,10 \pm 0,8$ & $1,10 \pm 0,9$ & 0,68 \\
\hline I.S. & & $0,64 \pm 0,2(36 \%)$ & $0,63 \pm 0,2(37 \%)$ & 0,26 \\
\hline $\begin{array}{l}\text { I.S. = índice de sucesso } \\
t=\text { teste de Student }\end{array}$ & & & & \\
\hline
\end{tabular}








Figura 3 - (Cagy) Análise vetorial agregada do astigmatismo induzido após o 60 mês de acompanhamento nos grupos a favor da regra e contra a regra. Os pontos escuros representam as médias e as elipses representam o desvio padrão. Cada anel do gráfico representa uma dioptria.

índice de sucesso apresentaram diferenças estatísticas entre os dois grupos no $1^{\circ}$ e $6^{\circ}$ de pós-operatório sendo maior em ambos os parâmetros no grupo contra a regra (Tabela 5). O astigmatismo induzido agregado foi de $0,75 \pm 1,21 \mathrm{D} @ 89^{\circ}$ no grupo a favor da regra e 1,26 $\pm 1,40 \mathrm{D} @ 3^{\circ}$ no grupo contra a regra (Figura 3).

\section{DISCUSSÃO}

Desde o início da cirurgia refracional, as incisões corneanas eram utilizadas para a redução do astigmatismo pré-operatório durante a cirurgia de catarata ${ }^{(13-16)}$. Tanto a utilização de incisões transversas, incisões arqueadas próximas ao centro da córnea, incisões corneanas perfurantes opostas à incisão principal e o aumento da incisão corneana foram utilizadas para a redução do astigmatismo pré-existente ${ }^{(13-14,17-18)}$. As incisões localizadas próximas ao centro óptico corneano, causavam mudanças na curvatura corneana, no componente esférico e aparecimento de astigmatismos irregulares. A realização de outra incisão oposta a principal aumentava o risco de complicações
Tabela 4. Distribuição do número de pacientes e porcentagem por dioptrias no pré e pós-operatório do $6 \%$ mês

\begin{tabular}{|c|c|c|c|c|}
\hline \multirow[b]{3}{*}{ D } & \multicolumn{4}{|c|}{$n=103$} \\
\hline & \multicolumn{2}{|c|}{ Pré-operatório } & \multicolumn{2}{|c|}{ Pós-operatório } \\
\hline & $\mathbf{N}$ & $\%$ & $\mathrm{~N}$ & $\%$ \\
\hline $0-0,50 \mathrm{D}$ & 0 & $0 \%$ & 13 & $12,62 \%$ \\
\hline $0-1,00 \mathrm{D}$ & 6 & $5,82 \%$ & 52 & $50,48 \%$ \\
\hline $0-1,50 \mathrm{D}$ & 33 & $32,03 \%$ & 80 & $77,66 \%$ \\
\hline $0-2,00 \mathrm{D}$ & 61 & $59,22 \%$ & 90 & $87,37 \%$ \\
\hline $0-2,50 \mathrm{D}$ & 83 & $80,58 \%$ & 99 & $96,11 \%$ \\
\hline $0-3,00 \mathrm{D}$ & 93 & $90,29 \%$ & 101 & $98,05 \%$ \\
\hline$>3,00 \mathrm{D}$ & 103 & $100,00 \%$ & 103 & $100,00 \%$ \\
\hline \multicolumn{5}{|c|}{$\mathrm{D}=$ valor dióptrico topográfico; $\mathrm{N}=$ número de pacientes } \\
\hline
\end{tabular}

especialmente o risco de infecção por ser uma outra entrada na câmara anterior. $\mathrm{O}$ aumento da incisão após a realização da facoemulsificação para 4,5 mm e 5,5 mm obrigava a utilização de suturas e depois a remoção das mesmas para o controle do astigmatismo induzido.

A utilização das lentes intra-oculares tóricas apresentava como complicação a rotação da lente no saco capsular no pósoperatório com a mudança do eixo do astigmatismo e piora da acuidade visual, necessitando nova intervenção para o reposicionamento no meridiano correto $^{(3-6)}$.

Observando as desvantagens encontradas nas técnicas anteriores as incisões relaxantes limbares se mostraram como uma excelente opção para a redução do astigmatismo préexistente durante a cirurgia de catarata ${ }^{(19-22)}$.

Em nosso estudo prospectivo com análise após o $1^{\circ}$ e o $6^{\circ}$ mês de seguimento, demonstramos que as incisões relaxantes limbares reduziram significativamente os astigmatismos préexistentes a favor da regra e contra a regra. A porcentagem de olhos com astigmatismo igual a uma dioptria aumentou de $5,82 \%$ para $50,48 \%$ após o $6^{\circ}$ mês.

Optamos por estudar separadamente os astigmatismos a favor da regra dos astigmatismos contra a regra com o objetivo de analisar a eficácia e possível interferência da posição das incisões limbares nesses dois grupos. Após esses estudos os dois grupos foram comparados entre si.

Em ambos os grupos ocorreram redução estatisticamente significativa entre o astigmatismo médio no pré-operatório comparado com o $1^{\circ}$ e $6^{\circ}$ mês de pós-operatório demonstrando a eficácia da técnica, com resultados semelhantes aos trabalhos publicados anteriormente ${ }^{(21,23-26)}$. Ocorreu redução do ín- 


\begin{tabular}{|c|c|c|c|c|c|c|c|c|c|}
\hline \multicolumn{10}{|c|}{ Tabela 5. Astigmatismo a favor da regra $\mathrm{x}$ contra a regra } \\
\hline & \multicolumn{9}{|c|}{$n=103$} \\
\hline & \multicolumn{3}{|c|}{ Pré-operatório } & \multicolumn{3}{|c|}{ Pós- 1ำ mês } & \multicolumn{3}{|c|}{ Pós - 6 mês } \\
\hline & AFR & CR & $p$ & AFR & CR & $p$ & AFR & CR & $p$ \\
\hline Astigmatismo & $2,1 \pm 1,0$ & $2,2 \pm 0,9$ & 0,86 & $1,10 \pm 0,7$ & $1,30 \pm 0,6$ & 0,11 & $1,40 \pm 0,8$ & $1,10 \pm 0,7$ & 0,56 \\
\hline Indução & & & & $1,10 \pm 0,8$ & $1,90 \pm 0,9$ & $<0,00001$ & $1,10 \pm 0,9$ & $1,70 \pm 0,8$ & 0,0001 \\
\hline I.S. & & & & $37 \%$ & $50 \%$ & 0,0028 & $36 \%$ & $49 \%$ & 0,0008 \\
\hline
\end{tabular}

dice de sucesso e da indução entre o $1^{\circ}$ e o $6^{\circ}$ mês em ambos os grupos, porém sem significância estatística. Essa redução progressiva foi descrita em trabalhos anteriores ${ }^{(23-25)}$, tendo como explicação o processo de cicatrização das IRPL.

Comparados os dois grupos, os astigmatismos médios topográficos não apresentaram diferenças estatísticas no préoperatório, $1^{\circ}$ e $6^{\circ}$ mês. A indução e o índice de sucesso apresentaram diferenças estatisticamente significativas entre o $1^{\circ}$ e o $6^{\circ}$ mês, com valores de menor indução e índice de sucesso no grupo a favor da regra. Esse resultado pode ser explicado pela ação também relaxante da incisão utilizada na facoemulsificação. Sendo ela temporal, agiria aumentando a aplanação no meridiano horizontal e, ao contrário, aumentando a curvatura no meridiano vertical, causando aumento do efeito corretivo nos astigmatismo contra a regra e diminuindo nos astigmatismos a favor da regra ${ }^{(21,23-26)}$.

No nomograma utilizado, a idade é fator determinante para o tamanho da incisão. A regressão linear mostrou não haver associação entre idade do paciente e astigmatismo induzido, o que nos leva a concluir que o nomograma é corretamente ajustado para o parâmetro idade.

A análise vetorial agregada do astigmatismo mostrou diminuição entre o pré e pós-operatório em ambos os grupos estudados, porém com pequena mudança no meridiano inicial do astigmatismo (quatro graus no grupo a favor da regra e cinco graus no grupo contra a regra). Esse achado sugere uma correta localização das incisões quando utilizada a topografia computorizada como base para o posicionamento das incisões nos meridianos mais curvos ${ }^{(26)}$.

Em nosso estudo não tivemos nenhuma perfuração corneana, sugerindo uma boa segurança nos bisturis de diamantes pré setados em $600 \mu$.

No trabalho inicial descrito do Nichami, ele preconizava a realização das incisões no final da cirurgia ${ }^{(8)}$. No nosso estudo realizamos as incisões no início, com o intuito de evitar o aumento da espessura corneana por hidratação e a hipotensão no final da cirurgia. Ambas as situações provocariam diminuição da profundidade das incisões e conseqüentemente menor efeito de aplanação ${ }^{(26)}$. Wang relata a possibilidade de menor correção no seu estudo ao fato das incisões terem sido realizadas ao término da cirurgia de catarata ${ }^{(21)}$.

Em conclusão, consideramos a técnica prática, simples, segura e efetiva na redução do astigmatismo pré-existente durante a cirurgia de catarata. A hipocorreção atingida em ambos os grupos estudados e principalmente no grupo a favor da regra sugere que novos estudos sejam realizados e que ajustes devam ser realizados no nomograma para serem atingidas melhores correções.

Com a entrada no mercado das lentes intra-oculares multifocais a diminuição do astigmatismo para valores iguais ou inferiores a 1,00 dioptria é importante para aumentar o número de pacientes candidatos para essa nova tecnologia. Com isso as incisões limbares relaxantes são sem dúvida uma das opções para a redução do astigmatismo pré-operatório.

\section{AGRADECIMENTOS}

Agradeço aos professores Maria Luiza Garcia Rosa e Mauricio Cagy ambos Professores Adjuntos na Cadeira de Epidemiologia da Universidade Federal Fluminense.

\section{ABSTRACT}

Purpose: To evaluate the effect of peripheral limbar relaxing incisions (PLRI) in the reduction of the astigmatism during cataract surgery. Methods: We studied prospectively 103 eyes of 103 patients submitted to PLRI, using the Nichamim nomogram during cataract surgery by phacoemulsification. After the first and sixth month we analized the changes in astigmatism topography, induction of astigmatism and sucess rate. The patients were divided into two groups according to the astigmatism (with-the-rule and against-the-rule), and studied separately. Results: There was a statistically significant change in the mean astigmatism topography in the preoperative and postoperative periods in both groups. There was an induction of $1.10 \pm 0.9 \mathrm{D}$ and $37 \%$ of sucess rate in the withthe-rule group and $1.70 \pm 0.80 \mathrm{D}$ and $51 \%$ of sucess rate in the against-the-rule group after six months of follow-up. Conclusions: The peripherical relaxing limbal incisions (PLRI) are effective in reducing the preoperative astigmatism during cataract surgery. The procedure is safe and easy. The Nichamin nomogram caused hypocorrection in both types of preoperative astigmatism.

Keywords: Astigmatism; Astigmatism/surgery; Phacoemulsification; Cataract extraction 


\section{REFERÊNCIAS}

1. Polisuk P, organizador. Topografia da córnea: atlas clínico. $2^{\underline{a}}$ ed. Rio de Janeiro: Cultura Médica; 2004.

2. Artola A, Ayala MJ, Claramonte P, Pérez-Santonja JJ, Alió JL. Photorefractive keratectomy for residual myopia after cataract surgery. J Cataract Refract Surg. 1999;25(11):1456-60.

3. Shimizu K, Misawa A, Suzuki Y. Toric intraocular lenses: correcting astigmatism while controlling axis shift. J Cataract Refract Surg. 1994;20(5):523-6.

4. Frohn A, Dick HB, Thiel HJ. Implantation of a toric poly(methyl methacrylate) intraocular lens to correct high astigmatism. J Cataract Refract Surg. 1999;25(12):1675-8.

5. Ruhswurm I, Scholz U, Zehetmayer M, Hanselmayer G, Vass C, Skorpik C. Astigmatism correction with a foldable toric intraocular lens in cataract patients. J Cataract Refract Surg. 2000;26(7):1022-7.

6. Sun XY, Vicary D, Montgomery P, Griffiths M. Toric intraocular lenses for correcting astigmatism in 130 eyes. Ophthalmology. 2000;107(9):1776-81; discussion 1781-2.

7. Gills JP. Treating astigmatism at the time of cataract surgery. Curr Opin Ophthalmol. 2002;13(1):2-6.

8. Nichamin LD. Treating astigmatism at the time of cataract surgery. Curr Opin Ophthalmol. 2003;14(1):35-8.

9. Fine IH. Self-sealing corneal tunnel incision for small-incision cataract surgery. Ocular Surg News. 1992; May 1:38-9.

10. Alpins NA. A new method of analyzing vectors for changes in astigmatism. J Cataract Refract Surg.1993;19(4):524-33. Comment in: J Cataract Refract Surg. 1994;20(3):366-7.

11. Alpins NA. New method of targeting vectors to treat astigmatism. J Cataract Refract Surg. 1997;23(1):65-75.

12. Holladay JT, Moram JR, Kezirian GM. Analysis of aggregate surgically induced refractive change, prediction error, and intraocular astigmatism. J Cataract Refract Surg. 2001;27(1):61-79.

13. Lindstrom RL, Agapitos PJ, Koch DD. Cataract surgery and astigmatic keratotomy. Int Ophthalmol Clin. 1994;34(2):145-64. Review.

14. Kershner RM. Keratolenticuloplasty: arcuate keratotomy for cataract surgery and astigmatism. J Cataract Refract Surg. 1995;21(3):274-7. Comment in: J Cataract Refract Surg. 1995;21(6):597-8.
15. Maloney WF, Grindle L, Sanders D, Pearcy D. Astigmatism control for the cataract surgeon: a comprehensive review of surgically tailored astigmatism reduction (STAR). J Cataract Refract Surg. 1989;15(1):45-54.

16. Maloney WF, Sanders DR, Pearcy DE. Astigmatic keratotomy to correct preexisting astigmatism in cataract patients. J Cataract Refract Surg. 1990;16(3):297-304

17. Lever J, Dahan E. Opposite clear corneal incisions to correct pre-existing astigmatism in cataract surgery. J Cataract Refract Surg. 2000;26(6):803-5. Comment in: J Cataract Refract Surg. 2000;26(12):1697-8. J Cataract Refract Surg. 2000;26(6):789-90. J Cataract Refract Surg. 2001;27(1):7-8.

18. Rao SN, Konowal A, Murchison AE, Epstein RJ. Enlargement of the temporal clear corneal cataract incision to treat pre-existing astigmatism. J Refract Surg. 2002;18(4):463-7. Comment in: J Refract Surg. 2003;19(5):610; author reply 610 .

19. Budak K, Friedman NJ, Koch DD. Limbal relaxing incisions with cataract surgery. J Cataract Refract Surg. 1998;24(4):503-8.

20. Müller-Jensen K, Fischer P, Siepe U. Limbal relaxing incisions to correct astigmatism in clear corneal cataract surgery. J Refract Surg. 1999;15(5):586-9.

21. Wang L, Misra M, Koch DD. Peripheral corneal relaxing incisions combined with cataract surgery. J Cataract Refract Surg. 2003;29(4):712-22.

22. Kaufmann C, Petre J, Ooi K, Phipps S, Cooper P, Goggin M; The Queen Elizabeth Astigmatism Study Group. Limbal relaxing incisions versus onaxis incisions to reduce corneal astigmatism at the time of cataract surgery. $\mathrm{J}$ Cataract Refract Surg. 2005, 31(12):2261-5. Comment in: J Cataract Refract Surg. 2006;32(9):1408; author reply 1408 .

23. Gimbel HV, Sun R. Postoperative astigmatism following phacoemulsification with sutured vs. unsutured wounds. Can J Ophthalmol. 1993;28(6):259-62.

24. Huang FC, Tseng SH. Comparison of surgically induced astigmatism after sutureless temporal clear corneal and scleral frown incisions. J Cataract Refract Surg. 1998;24(4):477-81.

25. Gills JP, Sanders DR. Use of small incisions to control induced astigmatism and inflammation following cataract surgery. J Cataract Refract Surg. 1991:17 Suppl:740-4.

26. Carvalho MJ. Avaliação da correção do astigmatismo corneano com incisão relaxante limbar na cirurgia extracapsular da catarata pelo método da facoemulsificação [tese]. São Paulo: Universidade Federal de São Paulo. Escola Paulista de Medicina; 2003. 